\title{
Estructura mediática y neoconservadurismo: Rupert Murdoch y su desembarco en EE.UU.
}

\author{
Lorena Romero DOMÍnguez \\ Universidad de Sevilla \\ lorenaromero@us.es \\ Antonio PINEDA CACHERO \\ Universidad de Sevilla \\ apc@us.es
}

Recibido: 29/03/2010

Aceptado: 21/11/2010

\begin{abstract}
Resumen
Este artículo analiza el desembarco mediático del magnate de la comunicación Rupert Murdoch en Estados Unidos, prestando atención a las relaciones establecidas con el movimiento conservador y el Partido Republicano, y a las repercusiones de esta conexión sobre el panorama ideológico de los medios de comunicación estadounidenses. Se tienen en cuenta los antecedentes empresariales de Murdoch, así como su procedencia y el proceso de adquisición de medios de comunicación en Estados Unidos. También analizamos la forma en que ciertos medios de Murdoch han funcionado como una voz mediática para ciertas facciones de la derecha estadounidense, siendo el canal de televisión Fox News paradigmático al respecto. El apoyo de Murdoch a candidatos republicanos en las elecciones presidenciales de 2000, 2004 y 2008 se considera una extensión política de su apoyo ideológico a la derecha.

Palabras clave: Estructura mediática, Estados Unidos, Medios conservadores, Partido Republicano, Fox News.

\section{Media Structure and Neoconservatism: Rupert Murdoch and his Media Arrival in the USA}

\begin{abstract}
This paper analyzes the media arrival of media mogul Rupert Murdoch in the United States. We focus on the ties with the American conservative movement and the Republican Party, and on those ties' consequences regarding the ideological landscape of American media. Murdoch's business past is taken into account, as well as the country Murdoch comes from, and the process of media acquisition in the United States. We also focus on the way certain media owned by Murdoch have worked as a media voice for certain factions of the American right. In this regard, Fox News Channel can be cited as a paradigmatic mass medium. Murdoch's support for Republican candidates in the presidential elections of 2000, 2004, and 2008, is regarded as a political extension of his ideological support for the right-wing.
\end{abstract}

Key words: Media structure, United States, Conservative media, Republican Party, Fox News.

\section{Referencia normalizada}

ROMERO DOMÍNGUEZ, Lorena y PINEDA CACHERO (2011): "Estructura mediática y neoconservadurismo: Rupert Murdoch y su desembarco en EE.UU”. Estudios sobre el mensaje periodístico. Vol. 17, núm. 1, págs.: 195-214. Madrid, Servicio de Publicaciones de la Universidad Complutense.

Sumario: 1. Introducción. 2. Desarrollo del estudio. 2.1. El imperio de Rupert Murdoch. 2.2. El sueño americano: el desembarco mediático de Murdoch en EE.UU. 2.3. Estructura mediática y relaciones políticas. 2.4. El conservadurismo del australo-estadounidense Murdoch: estructura mediática y relaciones políticas en Estados Unidos. 2.5. Una voz mediática para la derecha conservadora estadounidense. 2.5.1. News Corporation y el movimiento conservador. 2.5.2. El caso de Fox News Channel. 2.6. News Corporation y el apoyo político-electoral al Partido Republicano. 3. Conclusiones. 4. Referencias Bibliográficas. 


\section{Introducción}

Este trabajo se basa en un análisis detenido del desembarco del magnate de la comunicación Rupert Murdoch y su megacorporación mediática News Corporation en Estados Unidos. El objetivo fundamental del artículo es estudiar las conexiones existentes entre propiedad mediática, orientaciones ideológicas de los medios de comunicación, y apoyos a partidos políticos. Para ello, se tienen en cuenta los antecedentes empresariales del magnate, su procedencia y el proceso de adquisición de medios de comunicación en Estados Unidos, así como la manera en que los medios de Murdoch han incidido sobre el panorama ideológico y político de los media estadounidenses. Con ese fin, nos basamos fundamentalmente en el discurso de Fox News Channel y su significación para el movimiento conservador. Otro objetivo de este artículo, directamente relacionado con el anterior, es contribuir al estudio de las relaciones existentes entre la estructura mediática de la comunicación y el apoyo político en época de elecciones; en el caso de Murdoch, estas relaciones se ejemplifican mediante las conexiones establecidas con el Partido Republicano y el apoyo brindado a candidatos de este partido en las elecciones presidenciales de 2000, 2004 y 2008.

Como hipótesis de trabajo, consideramos que el asentamiento de News Corporation en Estados Unidos supone un caso modélico de las relaciones existentes entre la línea editorial de los grandes grupos de comunicación y el posicionamiento en un mercado de medios a través de variables ideológicas, las cuales coexisten con motivaciones puramente empresariales. Por ello, la atención al factor ideológico de los movimientos mediáticos de Murdoch gravitará sobre nuestro análisis desde un punto de vista metodológico.

\section{Desarrollo del estudio}

\subsection{El imperio de Rupert Murdoch}

En marzo de 2008, la revista Der Spiegel dedicaba un especial de tres páginas a los infructuosos intentos del magnate Rupert Murdoch de hacerse un hueco en el mercado televisivo alemán a través de la adquisición de la cadena de pago Premiere. En su presentación del imperio mediático australiano (cfr. HÜLSEN, 2008), el semanario editado por Spiegel Verlag era tajante al manifestar el fuerte carácter expansivo del grupo de Murdoch, cuyo retrato en la publicación alemana era el de un estoico conquistador que, país tras país, había conseguido que sus cadenas de televisión y sus periódicos estuvieran presentes en todo el orbe, desde Pekín hasta Los Ángeles, independientemente del régimen, gobierno, partido político o ideología dominante en cada uno de ellos. Una constatación plasmada también por Alan Knight en Media Globe: Trends in international Mass Media: "News Corporation became the first fully globalized media consortium by detaching itself from the vestiges of its Australian corporate origins in 2004 and locating itself squarely in the top of five hundred U.S. corporations" (KNIGHT, 2006: 119).

En menos de cincuenta años, el imperio familiar de Murdoch había conseguido convertirse en un gigante multimedia encargado de vender no solo noticias, sino una cultura corporativa y una ideología. Primero en Australia, donde el joven Murdoch 
se puso al frente de la Herald \& Weekly Times Limited con tan sólo 22 años, tras la muerte de su padre, y eliminó cualquier título que hiciera competencia a la herencia periodística legada por su progenitor (por ejemplo, la efectuada por John Fairfax e hijos). Después, en Reino Unido, cuyo desembarco se produjo en 1968 a través de la adquisición de News of the World, de la familia Carr, y al que TucCILle (2003: 23) define como "the grandaddy of Sunday tabloid journalism". Seis meses después era ya el director del periódico ${ }^{1}$ y en 1969 se hacía con The Sun. La paleta de periódicos sensacionalistas británicos se completaba en la década de 1980 con los "títulos serios" de la escena periodística británica, The Times y Sunday Times, tras la autorización, no exenta de polémicas como indica Aurora LABIo (cfr. 2008), de la primera ministra Margaret Thatcher ${ }^{2}$. Estos tempranos éxitos del joven Murdoch, quien adquirió la conciencia de que la supervivencia sólo vendría de la mano de la transnacionalización de Herald \& Weekly Times Limited, despertaron su interés por Estados Unidos, adonde acudía con regularidad para comprar programas para las cadenas de televisión que había adquirido en las ciudades de Adelaida y Sydney. Según Timothy Marjoribanks, América emergía ante Murdoch, como para otros muchos inmigrantes, al modo de una tierra de oportunidades con suficiente capital para invertir en sus arriesgados negocios, con una posición aventajada en la primera línea de la innovación tecnológica y, sobre todo, con un mercado laboral poco intervenido por el Gobierno (a través de la Comisión Federal de Comunicaciones, FCC en adelante) y por los sindicatos (MARJORIBANKS, 2000: 164).

\subsection{El sueño americano: el desembarco mediático de Murdoch en EE.UU.}

Todas las circunstancias anteriormente citadas hacían de Estados Unidos un apetecible horizonte para la aventura mediática de Murdoch. Ayudaban a ella, además, otros factores de índole personal, como su amistad con Leonard Goldenson, presidente de la American Broadcasting Company (ABC), a quien TuCCILLE (2003: 20) califica de mentor de Murdoch en Estados Unidos, y las aspiraciones literarias de su mujer, Anna, quien mostraba su interés por la escena cultural estadounidense y, además, no soportaba la ciudad de Londres, donde residía la familia Murdoch. La

\footnotetext{
${ }^{1}$ Periódico en el cual también tenía un interés ideológico, pues con su adquisición pretendía frustrar los intentos del político laborista Robert Maxwell de adquirir el diario para sus intereses partidistas.

${ }^{2}$ Aunque no es el objetivo fundamental de nuestro artículo, el caso de Murdoch también puede ser interesante para testar la teoría de que las condiciones estructurales de propiedad de los medios están vinculadas bidireccionalmente con apoyos políticos. Según Aurora LABIO BERNAL, los grandes grupos de comunicación “... van a apoyarse en la clase política del país para aumentar sus negocios aunque ello pueda incluir una posición dominante en el mercado o pueda poner en peligro la libertad de información. La estrategia seguida por los políticos va a ser la permisividad en la concentración de medios en manos de unas pocas empresas y la tendencia a beneficiar siempre a los mismos en la concesión de licencias o en la falta de obstáculos para seguir creciendo. Después habrá que pasar por caja y las ramificaciones mediáticas de los grupos tendrán que devolver el favor en forma de apoyo incondicional hacia el candidato en cuestión, que encontrará así a su servicio unos impagables órganos propagandísticos” (LABIO BERNAL, 2006: 77).
} 
suma de estas variables convertía en inevitable la expansión hacia el continente americano, una apertura condicionada por la propia filosofía empresarial del aussie y, según la cual, la única fuerza que podría frenar su carrera expansiva sería la de la muerte, "the only good regulator" (COMPAINE y GOMERY, 2000: 238).

Sin embargo, a pesar de las altas expectativas de Murdoch, el magnate llegaba a los Estados Unidos en un momento delicado, periodísticamente hablando, pues las ventas de las principales cabeceras habían caído drásticamente y los diarios, incluidos los grandes títulos como Herald Tribune o Daily Mirror, estaban inmersos en una vorágine de fusiones y cierres que podría truncar el triunfante desembarco del australiano. Quizá por esa razón el empresario decidiera iniciar su «sueño americano» asumiendo la responsabilidad de dos pequeñas publicaciones en San Antonio, Texas, donde Murdoch se hacía cargo de un modesto periódico local, el News, propiedad de la cadena Harte-Hanks, que trataba de competir con el Light del poderoso Hearst. Una lucha en franca desventaja si atendemos a la circulación de ambas cabeceras: 60.000 frente a 120.000, respectivamente. A pesar de ello, la estrategia empleada por Murdoch para revitalizar el News (tabloidizar la publicación con contenidos centrados en aspectos morbosos, como ya había hecho con el "affaire Keeler"3 en el británico News of the World) generó una leve subida a 75.000 lectores que, aunque imperceptible en el mercado estadounidense, se convertía en el aval necesario para emprender su siguiente obra: la creación de un gran periódico nacional, del tipo del National Enquirer, cuya circulación ascendía a cinco millones de ejemplares, pero reafirmando los rasgos sensacionalistas introducidos en el News, tanto en el británico como en el tejano.

El resultado fue la creación del National Star, conocido popularmente como el Star, un título que, a pesar de sus cuantiosas pérdidas (le había costado más de 12 millones de dólares iniciar su andadura), le permitiría entrar en contacto -gracias a las gestiones de su amigo Goldenson y sus compañeros de la administración Nixoncon la elite económica y periodística del país. Entre ellos se encontraba la propietaria del siguiente de sus objetivos periodísticos, Dorothy Schiff, dueña del deseado New York Post. Con las gestiones iniciadas para hacerse con esta cabecera se ponía la siguiente piedra en la construcción del gran imperio News Corporation. Una empresa, no obstante, compleja porque la propietaria del periódico no estaba dispuesta a sucumbir a las transformaciones ideológicas que acarreaba dejar su histórico periódico en manos de Murdoch, quien ya había evidenciado en Australia y en Reino Unido sus inclinaciones hacia la política conservadora (cfr. LABIO BERNAL, 2008). Tras unas duras negociaciones el trato se cerró en 1976 y Murdoch acabó al frente de un rotativo que, tras su consecuente transformación ideológica y temática, se presentaba ante la opinión pública como un híbrido entre el Star y el Enquirer. Gracias a la nueva fórmula periodística, las tiradas se duplicaron, alcanzando las 900.000 copias. Ello significaba que, a pesar de los detractores que

\footnotetext{
${ }^{3}$ Murdoch se decidía a publicar las polémicas memorias de Christine Keeler, la prostituta que había mantenido una relación simultánea con el Ministro de Defensa británico, John Profumo, y con un espía ruso. El conocimiento del idilio provocó la destitución de Profumo y la caída del gobierno conservador de Harold Macmillan en 1963.
} 
el magnate empezaba a tener en suelo norteamericano (por el "amarillismo" que imprimía a sus periódicos), estaba claro que el público en nada coincidía con la línea de los críticos. De este modo, Murdoch conseguía hacerse con un amplio sector de población al que le interesaban las proclamas del nuevo diario que, cada vez más, se escoraba hacia esa derecha política de la que había recelado su antigua propietaria. Como apunta TuCCILle (2003: 55), "The Post was totally renovated into a rightwing tabloid”, y gracias a ello era capaz de rellenar un hueco existente en el mercado de los medios estadounidenses como era la opinión pública de derechas. Un apetecible sector al que Murdoch ofrecería también las tribunas del Boston Herald, del Chicago Sun Times, y del Village Voice y el New West, tras su alianza con Clay Felker, de la New York Magazine Company. El vacío (o, al menos, la escasez) de medios alineados con la derecha en Estados Unidos se convertía, de este modo, en una veta de inigualable valor dispuesta a ser explotada por el australiano en su expansión transnacional, y será el mismo camino que se seguirá con la creación de Fox News Channel, como veremos más adelante.

\subsection{Estructura mediática y relaciones políticas}

Este vuelco hacia la derecha operado en todas sus posesiones mediáticas se producía tras renegar Murdoch de la ideología comunista, tendencia que había profesado en la Universidad y gracias a la cual se le había conocido como Rupert El Rojo ${ }^{4}$. Desde entonces Murdoch no había ocultado en ningún momento sus "inclinaciones” hacia las fuerzas conservadoras, pero más importante aún, no escondía que esos "coqueteos" con la clase política obedecían a un fin puramente económico como era la consecución de un trato de favor para sus negocios o, en todo caso, que la administración correspondiente le dejara hacer por omisión de sus obligaciones. Así lo ha reconocido la profesora Aurora LABIO (2008): "Realmente, Rupert Murdoch se debe a su corporación y sus vaivenes ideológicos estarán motivados por los intereses que de manera más segura beneficien su crecimiento y expansión”. Una actitud llevada al extremo, como apuntaba Joe Conason en The New York Observer tras el anuncio de adquisición del Wall Street Journal realizado por Murdoch: “The beneficiaries have spanned the ideological spectrum, from the right-wing Margaret Thatcher to the Marxist-Leninist daughter of Deng Xiaoping. All they had in common was their capacity to advance the interests of News Corp" (CONASON, 2007: web).

Esta historia de oscilaciones ideológicas en función de sus intereses económicos es la estrategia que parece haber aplicado Murdoch desde sus comienzos en Australia. Así, por ejemplo, podemos citar el trato de favor recibido desde la administración laborista de Bob Hawke, cuando el primer ministro declinó las peticiones de los periodistas para endurecer la legislación anti-concentración y conseguir frenar, con ello, el avance periodístico de Murdoch en la década de 1980 (KNIGHT, 2006: 120). La edición del 18 de enero de 1987 de The New York Times retrataba el modus operandi financiero de Murdoch y sus contactos con Hawke:

\footnotetext{
${ }^{4}$ Para la evolución ideológica de Murdoch, véase McKNIGHT, 2003.
} 
“The \$1.6 billion takeover by Mr. Murdoch has also shown a surprisingly symbiotic relationship between the Labor Government of Prime Minister Bob Hawke and the conservative publisher. The ruling Labor Party declined to interfere with Mr. Murdoch's acquisition of the 142 newspapers, radio and television stations owned by The Herald and Weekly Times. Mr. Murdoch has agreed to sell some of the properties, but he would nonetheless be left with 65.8 percent of the national readership” (PERLEz, 1987: web).

Y ello sucedía después de que Murdoch hubiera orquestado la campaña periodística para derribar al anterior laborista en el poder, Gough Whitlam, en 1975, cuando sus periódicos lanzaron una dura campaña difamatoria (escándalos sexuales incluidos) contra quien había negado al empresario australiano la explotación de una mina de bauxita en el oeste del país. Para Murdoch, sin embargo, la razón del desencuentro con Whitlam se debía a la negativa del gobierno australiano de permitir la entrada de barcos norteamericanos como señal de rechazo por la guerra de Vietnam. El líder laborista se distanciaba de la política bélica de Nixon sin saber que Murdoch se dejaba seducir por la gestión del presidente estadounidense y empezaba a operar un necesario viraje ideológico para convertir en realidad las esperanzas depositadas en el mercado norteamericano; un mercado donde, a nuestro juicio, el posicionamiento ideológico de News Corporation ha sido más claro y estable.

En cualquier caso, el juego al ratón y al gato con las autoridades políticas -como lo ha definido Jack Waterford, director del Canberra Times- en Australia, en Reino Unido y posteriormente en Estados Unidos, siempre generaba un sustancioso beneficio a las empresas del magnate: "Murdoch established a pattern of exercising editorial support for politicians who in turn favours News priorities. He was unique, in that he had the drive, discipline, and coordination to spread this practice across the globe” (WATERFORD, 2004: web).

Aunque, como afirma KNIGHT (2006: 119), Murdoch no parece haber hecho nada que no hubiera realizado su predecesor en Australia, donde el padre del magnate se había vinculado con políticos conservadores para favorecer sus negocios, llegando a conseguir, incluso, la condecoración de Sir. Siguiendo el ejemplo paterno, Murdoch también se había servido de las autoridades australianas a su antojo para erradicar cualquier vestigio de la competencia que presentaban los empresarios Fairfax, propietario del Morning Herald y del Evening Sun, Packer, con el Daily Telegraph y el Sunday Telegraph, y Norton con el Mirror.

\subsection{El conservadurismo del australo-estadounidense Murdoch: estructura mediática y relaciones políticas en Estados Unidos.Estructura mediática y relaciones políticas}

Si esto había pasado en su país de origen, en Reino Unido la alianza la había establecido hasta 1997 con los tories, quienes allanaron la entrada en el mercado mediático del australiano. Siguiendo esta evolución, en Estados Unidos la relación natural caía del lado de la administración Nixon, por las ayudas que se esperaban recibir para el desarrollo de sus negocios. Además, si en el contexto del escándalo Watergate el entonces presidente se veía obligado a dimitir, Murdoch había confesado su deseo de ayudarle poniendo a su disposición un medio de comunicación 
conservador en el cual se relataran los ataques proferidos desde los órganos liberales ${ }^{5}$ (TuCCILle, 2003: 43-44). No debemos ocultar que en este acercamiento a Nixon también había tenido un papel determinante su amigo Goldenson, cercano a la Casa Blanca y a las elites económicas del país. En cualquier caso, parece evidente que desde muy temprano Murdoch había mostrado simpatías hacia la derecha estadounidense, y que pretendía orientar a sus medios en esa dirección.

Nixon sería el primero de una larga lista de contactos con los conservadores, a los que seguiría Ronald Reagan, quien le ayudó en su proceso de nacionalización como ciudadano norteamericano (a través de la consecución de la green card) para permitirle la entrada en el negocio televisivo en su país de adopción. Esto podría interpretarse como una compensación por haberle apoyado desde sus periódicos hacia la Presidencia en 1981. Pero necesitaría también la ayuda de su nuevo aliado político para derogar la normativa que le impedía compatibilizar sus cadenas con los periódicos que ya poseía en las ciudades de Nueva York y Boston. Esto sucedía en virtud de una ley aprobada en 1958, y esgrimida en este momento por la FCC, según la cual un empresario con intereses en medios impresos no podía cruzarlos con cadenas de televisión en la misma zona geográfica. Desde estos supuestos, su amistad con Reagan se orientaría ahora a minimizar el impacto de este régimen de incompatibilidades en el imperio australoestadounidense. La razón última para los denodados esfuerzos de Murdoch era bien sencilla: los obstáculos puestos por la FCC frenaban el ambicioso objetivo de crear una cadena de televisión que pudiera hacer frente a la ABC, la NBC y la CBS. Una empresa que, como señalan COMPAINE y GOMERY, nadie había sido capaz de realizar en 30 años (cfr. 2000: 213) hasta que Murdoch consiguió, por ejemplo, arrebatar los derechos televisivos de la Liga de Fútbol Americano a la CBS y, con posterioridad, hacer frente a la CNN de Turner con su propio espacio informativo, el canal Fox News, en el cual nos detendremos más adelante.

Si había conseguido que la derecha norteamericana se identificara con sus periódicos, le tocaba ahora el turno a la televisión. Con la experiencia adquirida en suelo británico a través de la BSkyB, donde la permisividad del gobierno de Margaret Thatcher fue, nuevamente, decisiva, el magnate australiano preparaba la creación de su cadena con la adquisición de la mitad de la histórica 20th Century Fox, en 1984, la Fox Broadcasting, un año después, y la compañía Metromedia, que poseía siete estaciones de televisión, dos años más tarde. Tan exitosos comienzos se vieron truncados, sin embargo, cuando entró en escena Edward “Ted” Kennedy, una de las numerosas víctimas políticas a quien la carrera empresarial de Murdoch había dejado en el camino. Los hechos se remontaban a 1980, cuando el Post de Murdoch se decantó por Jimmy Carter, en detrimento de Kennedy, como candidato demócrata a la casa Blanca. Una ambigua relación que acabó con el apoyo de Murdoch al candidato republicano, Ronald Reagan (Frattini y Colías, 1996: 70) ${ }^{6}$, pero sin dejar

\footnotetext{
${ }^{5}$ Para evitar confusiones respecto al significado político del adjetivo liberal en el contexto europeo, especificaremos que en su acepción estadounidense liberal equivale ideológicamente a posturas de centro o centro-izquierda, mientras que en Europa el término se aplica a posiciones derechistas desde el punto de vista económico. En este trabajo utilizaremos el término en su acepción estadounidense.

${ }^{6}$ Para el apoyo del New York Post a Ronald Reagan en 1980, véase STEPHENS, 1982.
} 
pasar por alto los beneficios obtenidos de su breve idilio con Carter, como apunta Conason en The New York Observer:

"What Mr. Murdoch needed was a low-interest, government-backed loan to buy Boeing jets for an Australian airline he then owned. Three days after they met at the White House, the New York Post published a rousing front-page editorial endorsement of Mr. Carter -and the following week, Mr. Murdoch's airline received a \$300 million corporate-welfare check from the U.S. Export-Import Bank” (CONASON, 2007: web).

Durante tres décadas, en tres continentes diferentes, Murdoch había empleado esta estrategia de "mantenerse fiel a todos los ministerios" para incrementar sus ingresos. Pero el empeño del Senador Kennedy consiguió hacerle vender sus periódicos de Nueva York y Chicago, así como la estación de televisión que ya había comprado en Boston. Kennedy había podido disfrutar, al final, de su venganza, pero Murdoch no estaba dispuesto a renunciar a convertirse en uno de los grandes en el mundo de la comunicación estadounidense y empleó sus contactos con Reagan para conseguir que la FCC cerrara la investigación abierta sobre 20th Holdings Corp., una subsidiaria de News Corporation a la que Murdoch había puesto al frente de sus cadenas de televisión para evitar problemas legales. Curiosamente, como señala Russ BAKER (cfr. 1998), la actitud de los republicanos conservadores, quienes siempre habían manifestado sus recelos hacia la entrada de empresarios extranjeros, consistió en atacar el carácter persecutorio de la FCC contra Murdoch. Superado el obstáculo de la Comisión (acabó reconociendo que en todo el proceso no se había infringido ninguna normativa), nacionalizado ya como ciudadano norteamericano, con la potente combinación resultante de unos estudios de cine y una cadena de televisión, Murdoch encontraba el camino despejado para la puesta en funcionamiento de la Fox Broadcasting Company.

\subsection{Una voz mediática para la derecha conservadora estadounidense}

Hay que especificar que, aunque Fox News Channel sea probablemente el medio de News Corporation que más se ha destacado como portavoz de tendencias políticamente conservadoras (y electoralmente republicanas), no es el único. Como hemos visto anteriormente, tampoco es el primer medio que Murdoch escora a la derecha para alimentar a ese mercado ideológico. En el contexto de la revolución conservadora que llevó a Ronald Reagan a la presidencia, los medios conservadores de Murdoch no operaban en un vacío absoluto. "El Wall Street Journal”, escribe Luis Ignacio LópEz (1988: 36) “fue en realidad el primer órgano de la gran prensa que adelantó y reflejó la revolución ideológica latente en la llegada de Ronald Reagan a Washington”. Por otro lado, y desde 1984, se daba el fenómeno radiofónico de Rush Limbaugh, un locutor de ascendencia republicana y retórica anti-impuestos que hacia mediados de la década de 1990 llegaba semanalmente a 20 millones de personas a través de 660 emisoras (cfr. MicKLETHWAIT y WoOLDRIDGE, 2006: 153-154). En esa misma década, Murdoch acabaría de consolidar su aparato mediático al servicio de la derecha con Fox News Channel, un componente fundamental en las vinculaciones de News Corporation con el movimiento conservador de Estados Unidos. 


\subsubsection{News Corporation y el movimiento conservador}

Una de las proposiciones generales que David McKnight (cfr. 2003) señalaba a partir de su estudio del periódico The Australian, propiedad de Murdoch, es que "the influence of the owner of a mass media institution is important in explaining the reasons for its particular outlook, bias and so on" (McKniGHT, 2003: 355). Esta idea es aplicable asimismo al caso de las empresas mediáticas de Murdoch en Estados Unidos, donde la influencia de las creencias políticas del propietario es particularmente importante en tanto que factor explicativo de cuestiones como el sesgo ideológico de ciertos medios de News Corporation. En otros términos, el conservadurismo personal de Murdoch supondría un factor influyente en la línea editorial conservadora de sus medios. Según Gary C. WoOdWARD (2006: 34): "Many of its operations, ranking from Fox News to the New York Post, carry the politically conservative imprimatur of its CEO, Rupert Murdoch. While the company is not quite an ideological monolith, its news outlets often reflect the Murdoch trademark of faux populism and right-wing indignation". Las convicciones conservadoras de Murdoch, por consiguiente, pueden haber tenido un papel relevante en la sintonía con la derecha estadounidense. Esta idea se refuerza si se considera que News Corp. no sólo tiene la peculiar cualidad de ser “... una de las pocas megacorporaciones controladas por una sola persona...” (PoOley, 2007: 36); también es un grupo mediático donde las intervenciones del propietario tienen cierta relevancia. En 1982, un año después de comprar el londinense The Times, Murdoch dijo: “¿Por qué no puedo dar instrucciones al Times, si lo hago con todos mis otros diarios?” (MurdOCH, citado en BÉNILDE, 2007b: 26). Según Marie BÉNILDE (2007b: 26), "Murdoch controla un imperio mediático cuya característica es imponer a las redacciones una estricta obediencia, mezclando estrechamente intereses políticos, económicos y financieros”. En este contexto, es esperable que las "instrucciones" de Murdoch abarquen también la esfera ideológica y política. Sobre todo si se tiene en cuenta el profundo interés que Murdoch presenta por la política (cfr. McKnight, 2003: 348). Uno de sus ejecutivos, de hecho, le describió como "a frustrated politician. He can’t leave politics alone” (Ibid.).

En este contexto, ciertos medios de Murdoch en Estados Unidos van a establecer conexiones institucionales con el movimiento conservador. La propia Fox News se nutre "de los análisis suministrados por las fundaciones y los think tanks de derechas, que se ocupan de promocionar a sus analistas en las páginas de opinión de los grandes medios de comunicación” (MARCO, 2007: 209). Hay que tener en cuenta que el movimiento conservador engloba a una serie de instituciones destinadas a proveer de una ideología a la derecha estadounidense y promocionarla: Heritage Foundation, Cato Institute, American Enterprise Institute for Public Policy Research (muy vinculado con la primera Administración de George W. Bush), Manhattan Institute, Reason Foundation, etc. Como escribe David Brock:

"The most visible on-air FOX News personalities were political and ideological partisans of a character rarely seen in professional news organizations. David Asman, a daytime anchor, had come from the Wall Street Journal editorial page and the Manhattan Institute. Brit Hume of ABC News, anchor of an evening newscast, moonlighted as a freelance writer for the Far Right American Spectator and the neoconservative Weekly 
Standard. Tony Snow, a sometime anchor and until recently host of FOX's Sunday morning show, was a former editorial page editor of the Washington Times, a White House speechwriter for George H. W. Bush, and a substitute host for Rush Limbaugh. While anchoring on FOX, Snow penned an article for the Republican National Committee's magazine, Rising Tide, endorsing Bob Dole in 1996. Catherine Crier, who has since left the network, had been an elected Republican judge in Texas” (ВRоск, 2005: 317).

Las estrellas de Fox parecen, pues, proceder de órganos del movimiento conservador de Estados Unidos, cuando no directamente de las filas y los apoyos del republicanismo. A la lista de Brock podría añadirse que Roger Ailes, presidente de Fox News Channel, también contó para el canal con Fred Barnes, editor ejecutivo del citado The Weekly Standard (cfr. HicKey, 1998: 31). Murdoch, por cierto, también es dueño del Standard, y «pierde un millón de dólares al año con ella» (MARco, 2007: 183). La revista fue fundada en 1995, un año antes que Fox News, y «se ha convertido en la revista de referencia de la elite de derechas» (Ibíd.). Mediante publicaciones como ésta, el imperio mediático de Murdoch se relaciona con el amplio universo ideológico de los think tanks y las publicaciones de naturaleza ideológica del conservadurismo. De hecho, Murdoch ha formado parte del Board of Directors del Cato Institute, uno de los más importantes think tanks de tendencia libertaria derechista en Estados Unidos. The Weekly Standard pertenece a otra línea ideológica en la derecha, la de los neoconservadores. La misma línea de Norman Podhoretz y su influyente revista Commentary, que también han sido financiados por Murdoch (cfr. McKnight, 2003: 349).

\subsubsection{El caso de Fox News Channel}

Cuidadosamente relacionada con el Partido Republicano y con la experiencia adquirida durante diez años en suelo norteamericano como televisión convencional, Murdoch preveía que el futuro estaría en el cable y el satélite, y, más concretamente, en un canal de noticias 24 horas a través del cual poder seguir manteniendo la máxima, como indica Marie BÉNILDE (cfr. 2007a), de imponer una estricta línea editorial en sus medios, además de combinar sus intereses políticos y económicos. En este contexto hacía su aparición Fox News Channel, un instrumento de propaganda de la derecha (como Bill Clinton la había definido en una entrevista en la propia Fox el 25 de septiembre de 2006 -cfr. "Dean On President Clinton Standing Up To Right-Wing Propaganda on Fox News Sunday”, 2006-); un enemigo perfecto para aniquilar a la liberal CNN de Turner $^{7}$, y, al mismo tiempo, una máquina de hacer dinero presente en la actualidad en más de 85 millones de hogares estadounidenses. Un éxito logrado, como apuntaba El Mundo en su reportaje sobre el décimo aniversario de la cadena, gracias a la reinvención del concepto de canal informativo:

“Así, Fox News ha reinventado el concepto de cadena de noticias. La innovación más obvia es el formato. Si CNN convirtió en su seña de identidad su capacidad para conectar en directo con cualquier lugar del mundo -lo que la catapultó al estrellato durante la

7 También la MSNBC, tercera cadena de noticias en los Estados Unidos, propiedad de NBC y Microsoft. Junto con la CNN copan al público de centroizquierda estadounidense. 
Guerra del Golfo de 1991- Fox News ha transformado sus estudios en una especie de tertulias radiofónicas especializadas en información nacional, con un número muy inferior de conexiones en directo y muy pocas referencias a lo que pasa fuera de EEUU” (PARDO, 2006: web).

Innovaciones de formato aparte, en este artículo nos interesa particularmente su orientación ideológica. Y para ello, nada mejor que advertir que, en la puesta en funcionamiento de la cadena, Murdoch contó con la ayuda del republicano Roger Ailes, asesor de viejos amigos del australiano como Nixon y Reagan, de quienes ya se había servido para realizar su desembarco en los Estados Unidos, y de quienes serían dos de sus principales aliados en estos años pasados: George Bush padre y, posteriormente, hijo. A pesar de las explícitas declaraciones de independencia ideológica de la cadena (en su página web define su información como "fair and balanced”), la tendencia conservadora exhibida por Murdoch desde que se hiciera cargo del negocio familiar convertía al nuevo canal en la voz mediática de la derecha.

Fox News es destacable tanto por su contribución a un estilo peculiar de realizar periodismo televisivo (mezclando espectáculo, agresividad y política), como por la importancia que tuvo el elemento ideológico en su nacimiento. Fox News se lanzó en 1996, justo en el ecuador de la era de Bill Clinton como presidente, como un intento por parte de Murdoch de cubrir el espacio ideológico que la mayor parte de canales informativos estadounidenses dejaba a su derecha. Como señalaba una de las estrellas de Fox News, Brit Hume, en un reportaje publicado en 1998: "If you look at the number of conservatives in broadcasting altogether, there are almost none! There are an awful lot of people who would not admit they are liberals. But they are. They really are!” (HumE, citado en HickEy, 1998: 34). Esto puede explicar que algunos antiguos empleados se hayan quejado, como informaba Columbia Journalism Review, de “... attempting to cook the facts to make a story more palatable to right-of-center tastes” (HiCKEY, 1998: 34). Dada la naturaleza política del posicionamiento de Fox News en el mercado mediático, eslóganes de la cadena como "Fair and Balanced” o "We Report. You Decide.” (que podrían considerarse $a$ priori como irónicos dado el sesgo abierto del canal) adquieren una nueva significación: Fox News sería “Justa y Equilibrada” respecto a lo que se percibe e imputa como un sesgo liberal en los medios de comunicación mainstream. Así, y además de coincidir con las tendencias ideológicas conservadoras del propietario, Fox News evidencia un intento de cubrir mediáticamente un espacio ideológico.

Un estudio realizado acerca de la percepción de sesgo a través de titulares tomados de las páginas web de CNN y de Fox News (en los dos meses finales de las elecciones presidenciales de 2004) indicaba que en el caso de CNN existía la percepción de un sesgo más a la izquierda que en el de Fox News, si bien los titulares tomados de Fox News no fueron percibidos como excesivamente conservadores (cfr. WEATHERLY et. al., 2007: 91, 97-101). En todo caso, existen también indicios de que Fox News sí ha basculado hacia el partidismo conservador (cfr. Boehlert, 2007). En 1998, Columbia Journalism Review aportaba indicios empíricos sobre la ubicación del componente derechista en Fox News: 
"A close monitoring of the channel over several weeks indicates that the news segments tend to be straightforward, with little hint of political subtext except for stories the news editors feel the "mainstream" press has either downplayed or ignored. [...]"

"If Fox's collective news hole -small for an all-news cable channel- offers largely untilted coverage, its discussion programs regularly and unabashedly convey a right-ofcenter sensibility, sometimes subtle, at other times overt” (HiCKEY, 1998: 32).

En esta línea, el noticiario estrella de Fox News es The O’Reilly Factor, un espectáculo populista en el que Bill O’Reilly se enfrenta y ataca a representantes del establishment liberal (cfr. Micklethwait y WooldRidge, 2006: 216). El componente populista del show de O’Reilly tiene implicaciones interesantes para comprender la estrategia propagandística conservadora a la hora de conectar con la clase trabajadora:

“O’Reilly afirma resueltamente ser «apolítico», un portavoz de la gente corriente desencantada con el establishment en su conjunto. De hecho, es un conservador populista clásico: un producto de la clase media baja irlandesa (o de la clase trabajadora, si hay que creer la versátil versión a la baja de O’Reilly sobre sus orígenes) resentido por el trato de las élites liberales hacia gente como él, convertidos en meros instrumentos de su acción afirmativa y sus planes de transporte escolar. O’Reilly tiene un felpudo debajo de su escritorio con la cara de Hillary Clinton y afirma orgullosamente que el suyo es el «único programa desde el punto de vista de la clase trabajadora»" (MickLeTHWAit y WOOLDRIDGE, 2006: 216).

Fox News mezcla este populismo dirigido a la clase obrera con anticomunismo (cfr. Micklethwait y WoOldRidge, 2006: 216-217). Dan Kennedy hace un interesante resumen del discurso ideológico de la cadena:

"WHAT MAKES Fox News a success, I suspect, is that it provides a safe harbor for folks who are uncomfortable with complexity. Fox provides a narrative structure to the news. It's a dangerous world out there, full of terrorists, crime, and cop-kicking protesters. The Democrats want to tax away all your money and have forgotten the lessons of 9/11. George W. Bush will keep you safe. Liberals control the media, and Fox News is the only place where you'll learn the truth" (KENNEDY, 2004: web).

En perspectiva, Fox News Channel se ha convertido en uno de los factores que ha cambiado el sesgo del panorama mediático estadounidense, desde un punto de vista liberal a otro conservative:

"Durante décadas, los conservadores se lamentaron de que era imposible que la televisión difundiera su mensaje. Con George W. Bush, Fox News alcanzó la mayoría de edad al sustituir en 2002 a la CNN (o el Canal de Noticias Clinton, como la denominaban los conservadores) como el canal de noticias más popular y proporcionar al movimiento conservador una forma completamente nueva de difundir ideas” (MicKLETHWAIT y WoOLDRIDGE, 2006: 215).

Incluso, hay quien ha argumentado que el efecto Fox ha movido el propio panorama mediático hacia la derecha (cfr. KENNEDY, 2004: web). La marginación ideológica de la derecha conservadora, en cualquier caso, es cosa del pasado: los medios conservadores se han convertido en una fuerza pujante en Estados Unidos (cfr. Fresneda, 2003). En la actualidad, el arsenal de medios de la derecha también cuenta con el imperio de Alan Gottlieb (creador del Centro para la Defensa de la 
Libre Empresa), que incluye una red nacional de tertulias radiofónicas con más de 100 emisoras asociadas y varios sellos editoriales (cfr. Micklethwait y WoOLDRIDGE, 2006: 236-237) ${ }^{8}$. Y, de la misma forma que Murdoch posicionó Fox News como alternativa al sesgo centrista de otras televisiones, han surgido empresas mediáticas que han intentado ocupar el espacio a la derecha de los medios liberales, como ocurrió con el diario The New York Sun, que salió a la venta en abril de 2002 "como una alternativa conservadora al «New York Times»" (AFP, 2008: 104), y que cerró en 2008.

\subsection{News Corporation y el apoyo político-electoral al partido Republicano}

El movimiento conservador estadounidense va más allá del Partido Republicano, hasta el punto de que, en ocasiones, no tienen ni por qué coincidir. Así, desde un punto de vista ideológico ser un candidato del Partido Republicano no equivale necesariamente a ser aceptado como un candidato "conservador" por parte de la derecha. En realidad, Ronald Reagan, que llegó a la presidencia en 1980, fue el primer presidente salido del movimiento conservador. George W. Bush es otro de los republicanos apoyados por dicho movimiento. Con políticos como Bush cristaliza el compromiso de los medios de Murdoch con la causa conservadora, por un lado, y con la administración republicana, por otro. Fox News es un ejemplo de medio de comunicación que participa de esta conjunción de intereses. Como señalaba Robert Greenwald (productor/director del documental Outfoxed: Rupert Murdoch's War on Journalism), Fox News no es sólo un canal conservador. "Fox News is a Republican news network. It takes a Republican line, which is different from a conservative line.” (Robert Greenwald, citado en Kennedy, 2004: web). Para David Brock, que trabajó en la institución conservadora Heritage Foundation (cfr. BROcK, 2005: 3), FOX "is now recognized for what it is -namely a propaganda organ of the GOP-..." (BRock, 2005: $382^{9}$ ). Así, al punto de partida ideológico de Fox News se añade un componente activo desde el punto de vista político-electoral.

Como contexto general, señalaremos que las relaciones generales de Murdoch y News Corporation con los republicanos son bastante fluidas. Ya se ha mencionado, por ejemplo, el papel de Roger Ailes en la propaganda republicana de las últimas décadas. La elección de Ailes como director de Fox News implica per se una determinada orientación política; según Neil Hickey (1998: 33), Ailes es “... the only network news boss whose political colors are plainly visible...”. La cobertura proporcionada al republicanismo también es un factor contextual a tener en cuenta sobre la tendenciosidad de la cadena. Según Fairness \& Accuracy in Reporting, 50 de 56 invitados partidistas en el programa diario de Brit Hume (en un periodo de 5 meses) eran republicanos, y sólo 6 demócratas (cfr. BROcK, 2005: 324).

Desde el punto de vista electoral, Murdoch ya había intentado oponerse a los Demócratas cuatro años antes de iniciar Fox News: "Murdoch did what he could to

${ }^{8}$ Para las relaciones entre los medios de comunicación y la derecha estadounidense, véase Micklethwait y WoOLdRidge, 2006: 215-219, 368-369; y Marco, 2007: 200-215.

9 “GOP” son las siglas de Grand Old Party, expresión con la que suele conocerse al Partido Republicano de Estados Unidos. 
prevent Bill Clinton's election in 1992, including running stories in his British papers, which were then picked up and published stateside [...] about matters like Clinton's visit to Moscow during his college days” (BAKER, 1998: 53). E incluso en el aspecto electoral, el apoyo político de News Corporation y Fox News al republicanismo no se limita a las elecciones presidenciales. El New York Post atacó a la candidata a la vicepresidencia (en 1984) Geraldine Ferraro, y apoyó a Giuliani (cfr. BROCK, 2005: 173). Según BAKER (1998: 54): “Three years into Giuliani’s first term, veteran Village Voice political reporter Wayne Barrett asked Post editorial page editor Eric Breindel if the paper had run a single editorial critical of the administration; Breindel, he says, admitted it had not”. El New York Post describió a Hillary Rodham Clinton como una "rejected wife" (citado en BROCK, 2005: 173); y Fox News también ha atacado a la ex-primera dama (MARCo, 2007: 209). Por el contrario, Arnold Schwarzenegger (candidato republicano y gobernador de California) era objeto de las siguientes palabras del presentador de Fox Tony Snow: "He ran on values. He's a great story because he was unapologetic about loving his county.... We want a sense of America that is strong and unapologetic." (Tony SNow, citado en BROCK, 2005: 331).

Todo esto puede servir de contexto para explicar el apoyo electoral que los medios de Murdoch, y Fox News especialmente, han brindado a los candidatos del Partido Republicano en las elecciones de 2000, 2004 y 2008. Algo que no debe ser demasiado extraño si se consideran, por ejemplo, los términos en que un memorando editorial de Fox aconsejaba cubrir informativamente a Bush: "His political courage and tactical cunning are worth noting in our reporting throughout the day" (citado en BRock, 2005: 391).

News Corp. estuvo entre las primeras corporaciones de medios en cuanto a contribución a la campaña presidencial estadounidense de 2000, con casi 800.000 dólares (cfr. Segovia, 2006: 122). Pero existen indicios de que, en el caso particular del Partido Republicano, el imperio mediático de Murdoch “contribuyó” también de una forma algo menos material al triunfo de George W. Bush frente al candidato demócrata Al Gore. Según escribe Brock en The Republican Noise Machine: "The George W. Bush administration was the first in history to have a TV network rooting for it, a fusion of power that the White House used to its full propaganda potential. On the night of the 2000 election, FOX had been the first to declare Bush the victor. Within a few minutes, all the other networks followed suit” (ВRоск, 2005: 332). Para su Election Night Decision Desk, la cadena contrató a un primo de Bush, John ELLIS, quien había escrito: "I am loyal to my cousin, Governor George Bush of Texas. I put that loyalty ahead of my loyalty to anyone else outside my immediate family" (citado en Boehlert, 2000: web). Por otro lado, en la campaña de 2000 circuló la historia de que Bush había sido arrestado por conducir bebido en 1976 (cfr. BoeHlert, 2000). Eric Boehlert aporta varias citas de Fox News que indican que la cadena intentó quitarle importancia a dicho asunto. Reproducimos algunas de ellas. Para O'Reilly: "It is a non-issue in my opinion"; para la invitada Mara LIASSON: "I think it's going to have little effect on George W. Bush's chances for the White House. It's not a bombshell."; para el invitado Mort Kondracke: "I think this is a minor story." (citados en BoeHLeRT, 2004: web). El New York Post también se 
sumó a la campaña de los medios conservadores contra Al Gore bajo la acusación de “LIAR, LIAR” (citado en BROCK, 2005: 4).

En las elecciones de 2004, más del 70\% de los votantes de Bush escogieron Fox como fuente de noticias (cfr. BROCK, 2005: 381), lo cual refuerza la hipótesis sobre el sesgo partidista de la cadena. En esta ocasión, Fox News explotó la atribución al candidato del Partido Demócrata, John Kerry, de un carácter cambiante (flip-flopping). De hecho, un memorando editorial de Fox decía que Kerry estaba "starting to feel the heat for his flip-flopping voting record.” (citado en BROCK, 2005: 391). La cadena promovió además el buen momento en el que se encontraba la economía. Y uno de sus programas mostraba, bajo una imagen de Kerry, la pregunta “Jobs Killer?”. Un estudio del Center for Media and Public Affairs encontró que durante el punto álgido de la campaña electoral, sólo el $13 \%$ de los comentarios sobre Kerry por parte de los participantes en debates de Fox News eran positivos, mientras que en el caso de Bush se trataba del 50\% (cfr. BoEHLERT, 2007). El New York Post también pareció unirse a la estrategia anti-Kerry de la derecha política en las elecciones de 2004 (cfr. BROCK, 2005: 383-384). E incluso el propio Rupert Murdoch expresó su deseo de que Bush ganase las elecciones de noviembre de 2004: "Lo logrará sin problemas", dijo Murdoch (citado en BBC Mundo.com, 2004: web).

En 2008, y aunque de manera más atemperada que en 2000 y 2004, los apoyos mediáticos de News Corporation también parecen haber oscilado hacia el Partido Republicano (cfr. Romero Domínguez, Labio Bernal, y Pineda CACHERo, 2009): John McCain recibió el apoyo de The Weekly Standard y Fox News (esta última, además, desarrolló una smear campaign contra Barack Obama), y The New York Post, aunque apoyó en las elecciones primarias del Partido Demócrata a Obama, se decantó por McCain en las presidenciales. The Weekly Standard, por su parte, no sólo apoyó a McCain, sino que uno de los miembros del staff del Standard, Michael Goldfarb, fue nombrado Deputy Communications Director de la campaña de McCain (cfr. GreEnwald, 2008). Meses después del triunfo electoral de Obama, Murdoch en persona volvió a posicionarse en 2009 en relación al candidato: "Creo que se ve como un presidente del cambio y eso implica un Gobierno más grande. No ha hecho secretos de ello. Creo que eso es peligroso" (Murdoch, citado en FERNÁNDEZ, 2009: web); poco después, la Administración Obama afirmaba, mediante la directora de Comunicaciones de la Casa Blanca Anita Dunn, que Fox News "a menudo opera casi como el brazo de investigación o el vocero del Partido Republicano” (DunN, citada en RodRÍGUEZ, 2009: 101) ${ }^{10}$.

\footnotetext{
${ }^{10}$ Parecen existir indicios en años recientes de que, como se ha visto anteriormente en relación a otros periodos de la creación del imperio mediático de Murdoch, las condiciones estructurales de propiedad mediática están vinculadas recíprocamente con el apoyo político. En este sentido, y según Aurora Labio Bernal, el apoyo de Murdoch al Partido Republicano "le ha producido excelentes resultados y le ha servido para sacar ventaja a sus competidores en algunos terrenos, como el de la televisión” (2006: 75). Según la misma autora: “... News Corporation consiguió el visto bueno de la FCC en la adquisición de DirecTV gracias a que se aseguró el voto de tres de los miembros republicanos de la Comisión. Por este motivo, es perfectamente comprensible que en una reciente entrevista concedida en la televisión
} 


\section{Conclusiones}

El desembarco de Rupert Murdoch y News Corporation en Estados Unidos supone un caso revelador respecto a las relaciones establecidas entre estructura mediática y tendencias ideológico-políticas en el contenido de los medios de comunicación. Consideramos que el caso de los medios de Murdoch ejemplifica la noción de que los cambios en las ideas dominantes en una sociedad establecen una relación con la promoción de dichas ideas en los medios de comunicación de masas; en un mundo angloparlante empujado hacia la derecha neoliberal y/o neoconservadora desde las revoluciones de Reagan y Thatcher a finales de la década de 1970 y principios de la de 1980, el posicionamiento de medios de News Corporation, como Fox News Channel o The Weekly Standard, o la transformación de The New York Post, reflejan asimismo un cambio hacia la derecha del espectro ideológico que, además, ha encontrado un público en Estados Unidos.

Si se observa la historia de la propaganda (cfr. PizArRoso Quintero, 1993), no hay nada nuevo en la idea de que el periodismo se ponga al servicio del poder. Lo interesante del caso que nos ocupa es que se ha producido una convergencia de factores que han transformado a News Corporation (y a la cadena Fox News en particular) en un símbolo del sesgo ideológico y el partidismo político. Tales factores coincidentes son variados: en primer lugar, es destacable que el lanzamiento de Fox News se plantease según un posicionamiento de mercado de tipo ideológico; en segundo lugar, que las convicciones políticas personales de Murdoch (y su tendencia a intervenir en el contenido de sus medios) coincidiesen sustancialmente con el ámbito ideológico que pretendía cubrir Fox News; en tercer lugar, la coincidencia histórica de que, poco después de la creación de medios como The Weekly Standard y Fox News, un candidato republicano de tendencia conservadora ganara en dos ocasiones las elecciones presidenciales, pudiendo interpretarse el apoyo recibido por parte de News Corporation como una extensión políticoelectoral de la labor promocional del conservadurismo que ya se estaba realizando en la esfera puramente ideológica. Por consiguiente, puede concluirse que los medios de Murdoch han apoyado a la Nueva Derecha de Estados Unidos en dos niveles distintos: por un lado, en la esfera de las ideas, proporcionando un altavoz a los ideólogos conservadores; por otro, en la praxis política, ejerciendo una política editorial partidista respecto a la administración republicana.

En este sentido, consideramos necesario destacar la conclusión de que, frente a supuestos vaivenes y oscilaciones ideológicas acaecidos en otros momentos de su historia, los medios de Murdoch en Estados Unidos han funcionado como un altavoz mediático conservador particularmente persistente. Los intereses empresariales y

pública de Estados Unidos, Murdoch manifestara su intención de respaldar públicamente a un candidato republicano en las próximas elecciones de 2008” (LABIO BERNAL, 2006: 77). La adquisición de DirecTV va a proporcionarle a Murdoch la oportunidad de ampliar su alcance mediático: "In December 2003, by a 3-2 vote, the Federal Communications Commission gave Rupert Murdoch's News Corp. permission to buy control of DirecTV, the largest satellite operator in the United States, giving it a platform to launch new cable channels reaching eleven million subscribers” (BROCK, 2005: 173). 
táctico-políticos coexisten en la experiencia estadounidense de News Corporation con motivaciones ideológicas.

Murdoch ha contribuido a que el aislamiento mediático del movimiento conservador estadounidense sea un hecho del pasado. Lo que fue un hueco de mercado aprovechado por el magnate ahora se ha generalizado; la inteligencia de Murdoch ha consistido en anticiparse (salvo excepciones) a todo ello, haciendo converger intereses empresariales y convicciones ideológicas.

\section{Referencias bibliográficas}

AFP (2008): "Desaparece el diario «The New York Sun» por la crisis financiera", en $A B C$ (Madrid), 1 de octubre, sección TV y Comunicación (p. 104).

BAKER, Russ (1998): "Murdoch’s Mean Machine”, en Columbia Journalism Review, $\mathrm{n}^{0}$ 37, Iss. 1. New York, Columbia University's Graduate School of Journalism, pp. 51-56.

BBC Mundo.com (2004): “Rupert Murdoch apoya a Bush”, en BBC Mundo.com: http://news.bbc.co.uk/hi/spanish/international/newsid_3607000/3607549.stm [fecha de consulta: 22 de agosto de 2008].

BÉNILDE, Marie (2007a): "Concentración de medios de comunicación, ética y relaciones de poder. Murdoch: comprador y mercader de influencia”, en rebelión.org: http://www.rebelion.org/noticias/2007/7/54110.pdf [fecha de consulta: 7 de noviembre de 2008].

- (2007b): "La prensa en manos de depredadores”, en Le Monde Diplomatique (edición española), agosto (p. 26).

BOEHLERT, Eric (2000): "Fox guarding the henhouse", en Salon.com: http://archive.salon.com/politics/feature/2000/11/15/ellis/index.html [fecha de consulta: 10 de octubre de 2008].

- (2004): "Rewriting history", en Salon.com: http:/dir.salon.com/story/news/ feature/2004/07/23/fox_dui/ [fecha de consulta: 10 de octubre de 2008].

- (2007): “Fox News can’t take a punch”, en Media Matters for America: http://mediamatters.org/columns/200703130004 [fecha de consulta: 17 de marzo de 2010].

BROCK, David (2005): The Republican Noise Machine. New York, Three Rivers Press.

BROWN, Tina (2005): "Rupert Murdoch, Bending With the Wind", en washingtonpost.com: http://www.washingtonpost.com/wp dyn/content/article/2005/09/ 14/AR20050914026 [fecha de consulta: 22 de agosto de 2008].

COMPAINE, Benjamin M. y GOMERY, Douglas (2000): Who owns the Media? Competition and Concentration in the Mass Media Industry. London, Routledge.

CONASON, Joe (2007): "Beware the Promises of Murdoch", en The New York Observer: http://www.observer.com/2007/beware-promises-murdoch [fecha de consulta: 7 de noviembre de 2008]. 
"Dean On President Clinton Standing Up To Right-Wing Propaganda on Fox News Sunday” (2006), en The Democratic Party website: http://www.democrats.org/ a/2006/09/dean_on_preside_3.php [fecha de consulta: 15 de marzo de 2010]

FERNÁNDEZ, Eduardo (2009): "Rupert Murdoch cree que en una década 'casi todos los diarios' serán digitales”, elmundo.es (9 de junio): http://www.elmundo.es/elmundo/2009/06/09/comunicacion/1244550145.html [fecha de consulta: 10 de febrero de 2010]

FRESNEDA, Carlos (2003): “El nacimiento del 'periodismo patriótico””, en elmundo.es: http://www.elmundo.es/papel/2003/03/12/mundo/1354350.html [fecha de consulta: 19 de septiembre de 2008].

FRATTINI, Eric y COLÍAS, Yolanda (1996): Tiburones de la comunicación. Madrid, Ediciones Pirámide.

GREENWALD, Glenn (2008): “Newest McCain official: President has "near dictatorial powers"” (Print), en Salon.com (Jun. 02, 2008): http://www.salon.com/opinion/greenwald/2008/06/02/goldfarb/print.html [fecha de consulta: 12 de enero de 2009]

HERTZ, Noreena (2002): El poder en la sombra. Barcelona, Ed. Planeta, S. A.

HICKEY, Neil (1998): “Is Fox News Fair?”, en Columbia Journalism Review, n 36, Iss. 6. New York, Columbia University’s Graduate School of Journalism, pp. 3035.

HÜLSEN, Isabell (2008): “Operation Nachtangriff”, en Der Spiegel, 14 de enero (pp. 80-82).

KENNEDY, Dan (2004): “GOP-TV”, en The Boston Phoenix: http://bostonphoenix.com/boston/news_features/dont_quote_me/multipage/document [fecha de consulta: 18 de agosto de 2008].

KNIGHT, Alan (2006): “Australia: media and globalization”, en ARTZ, Lee y KAMALIPOUR, Yahia R. (ed.): The media globe: trends in international mass media. Lanham (Maryland), Rowman \& Littlefield, pp. 119-139.

LABIO BERNAL, Aurora (2006): Comunicación, periodismo y control informativo. Barcelona, Anthropos.

- (2008): “Medios y política en Gran Bretaña. O cómo los laboristas conquistaron al conservador Murdoch”. Comunicación presentada al I Congreso de la AEIC (Santiago de Compostela).

LÓPEZ, Luis Ignacio (1988): Adiós, Mr. Reagan. Barcelona, Ediciones B.

MARCO, José María (2007): La nueva revolución americana. Madrid, Ciudadela.

MARJORIBANKS, Timothy (2000): News Corporation, Technology and the Workplace: Global Strategies, Local Change. Cambridge, Cambridge University Press.

MCKNIGHT, David (2003): “““A World Hungry for a New Philosophy”: Rupert Murdoch and the rise of neo-liberalism”, en Journalism Studies, Volume 4, Number. 3. London, pp. 347-358. 
MICKLETHWAIT, John y WOOLDRIDGE, Adrian (2006): Una nación conservadora. Barcelona, Debate / Random House Mondadori.

PARDO, Pablo (2006): "Fox News, la cadena conservadora que derrotó a la CNN, cumple 10 años”, en El Mundo: http://www.elmundo.es/papel/2006/10/08/ comunicacion/2034800.html [fecha de consulta: 8 de noviembre de 2008].

PERLEZ, Jane (1987): "Murdoch Holding Issue in Australia", en The New York Times: http://query.nytimes.com/gst/fullpage.html?res=9B0DEED81031 F93BA25752C0A961948260 [fecha de consulta: 7 de noviembre de 2008].

PIZARROSO QUINTERO, Alejandro (1993): Historia de la propaganda. $2^{\mathrm{a}}$ edición, ampliada. Madrid, Eudema.

POOLEY, Eric (2007): “Estoy orgulloso de tener tantos enemigos”, en XLSemanal $A B C, 12$ al 18 de agosto, pp. 34-40.

QUIRÓS, Fernando (1995): “Las cadenas de la información norteamericanas”, en Cuadernos de Información y Comunicación, $\mathrm{n}^{\circ}$ 1. Madrid, Servicio de Publicaciones de la Universidad Complutense de Madrid, pp. 115-142.

- (1998): Estructura internacional de la información: el poder mediático en la era de la globalización. Madrid, Síntesis.

RODRÍGUEZ, Pedro (2009): “Obama contra Murdoch”, en ABC, 29 de octubre, sección Televisión y Radio (p. 101).

ROMERO DOMÍNGUEZ, Lorena R., LABIO BERNAL, Aurora y PINEDA CACHERO, Antonio (2009): "De Europa a Estados Unidos: News Corporation, discurso ideológico y contextos políticos en las democracias contemporáneas”, en Trípodos [ EXTRA 2009 ], V Congrés Internacional Comunicació i REALITAT / V Congreso Internacional Comunicación y realidad, 1. Barcelona, Facultat de Comunicació Blanquerna-Universitat Ramon Llull, pp. 39-46.

SEGOVIA, Ana Isabel (2001): La estructura de los medios de comunicación en Estados Unidos: análisis crítico del proceso de concentración multimedia. Tesis Doctoral, Facultad de Ciencias de la Información, Universidad Complutense de Madrid.

- (2006): "Imperialismo corporativo y concentración mediática. La preponderancia del primer filtro", en VÁZQUEZ, Miguel y SIERRA, Francisco (coord.): La construcción del consenso. Revisitando el modelo de propaganda de Noam Chomsky y Edward S. Herman. Madrid, Sirandaeditorial / Gestión editorial Visionnet, pp. 113-142.

STEPHENS, Mitchell (1982): “Clout: Murdoch’s Political Post”, en Columbia Journalism Review. New York, Columbia University's Graduate School of Journalism, pp. 44-46.

TUCCILLE, Jerome (2003): Rupert Murdoch, Creator of a Worldwide Media Empire. Washington, Beard Books.

WATERFORD, Jack (2004): "Flaws in the Murdoch Empire”, en Canberra Times: http://www.canberratimes.com.au/news/local/news/opinion/flaws-in-themurdoch-empire/716649.aspx [fecha de consulta: 8 de noviembre de 2008]. 
WEATHERLY, Jeffrey N., PETROS, Thomas V., CHRISTOPHERSON, Kimberly M., y HAUGEN, Erin N. (2007): "Perceptions of Political Bias in the Headlines of Two Major News Organizations", en The Harvard International Journal of Press/Politics, Volume 12, n $^{\circ}$ 2. Cambridge, pp. 91-104.

WOODWARD, Gary C. (2006): Center Stage: Media and the Performance of American Politics (Communication, Media and Politics). Lanham (Maryland), Rowman \& Littlefield. 